2016 Управление, вычислительная техника и информатика № 4 (37)

\title{
Г.Ш. Цициашвили
}

\section{ПУАССОНОВСКИЕ ПОТОКИ В СИСТЕМЕ С ПОВТОРНЫМ ОБСЛУЖИВАНИЕМ}

\begin{abstract}
Рассматриваются системы массового обслуживания с бесконечным числом каналов и повторным обслуживанием общего вида. Эти системы представляются в виде сетей с бесконечным числом каналов в узлах и имеют вид ориентированных деревьев. С помощью теоремы Бурке устанавливаются условия, когда потоки в сети являются пуассоновскими и независимыми.

Ключевые слова: многоканальная и бесконечно канальная системы массового обслуживания; винеровский процесс; число занятых каналов.
\end{abstract}

Системы массового обслуживания с повторным обслуживанием заявок вызывают большой интерес у специалистов по массовому обслуживанию $[1,2]$. Особый интерес в последние годы вызывают системы с повторным обслуживанием и бесконечным числом каналов [3, 4]. В этих работах инициируется построение достаточно общих моделей обслуживания в сетях с бесконечным числом каналов в каждом узле. Особенностью таких сетей является их представление в виде ориентированного дерева. При анализе этих сетей с пуассоновским входным потоком появляется необходимость использовать теорему Бурке и применять теоретико-графовые построения для определения независимости отдельных потоков сети. В настоящей работе предпринимается попытка решения подобных вопросов в наиболее общем виде.

\section{1. Потоки, выходящие из одного узла}

Рассмотрим бесконечно канальную систему массового обслуживания с пуассоновским входным потоком и показательным распределением времени обслуживания заявок. Полагаем, что каждая заявка после окончания обслуживания может быть повторно обслужена (рис. 1). Возможны самые различные протоколы работы такой системы, например, время повторного обслуживания может иметь распределение, отличающееся от времени первоначального обслуживания, число повторных обслуживаний может быть отличным от единицы и т.д.
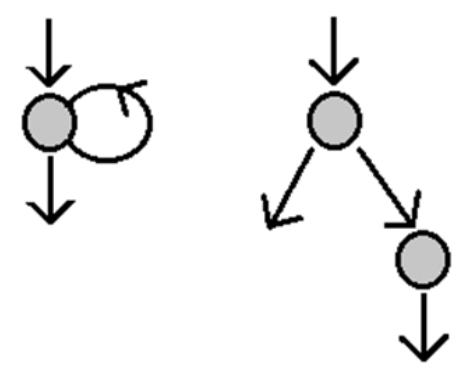

Рис. 1. Преобразование системы с однократным повторным обслуживанием в сеть

Самой общей может быть модель прохождения заявки через сеть, в каждом узле которой имеется бесконечное число каналов с показательным распределением времени обслуживания. Такая сеть описывается ориентированным деревом $D$, в котором каждое ребро направлено от корня дерева $r$ (вершины, в которую не входит направленное ребро) к его листьям (вершинам, из которых не выходят направленные ребра). После окончания обслуживания в узле заявка может быть направлена в один из 
узлов дерева, куда указывают его направленные ребра. Естественным обобщением этой модели является предположение, что время обслуживания заявки на приборе является гиперэкспоненциальным (вероятностной смесью экспоненциальных распределений). Данное обобщение никак не меняет вид сети, у которой вновь переходы заявок между узлами описываются ориентированным деревом.

Из известной теоремы Бурке [6] следует, что если сеть функционирует в стационарном режиме, то выходной поток из каждого узла, равно как и входной поток в каждый узел, является пуассоновским. Однако возникает вопрос о зависимости или независимости этих потоков.

Рассмотрим теперь пуассоновский поток точек $\Lambda=\left\{0 \leq t_{1} \leq t_{2} \leq \ldots\right\}$ интенсивности $\lambda$. Предположим, что каждая точка потока $\Lambda$ независимо с вероятностью $p_{1}, 0<p_{1}<1$, становится точкой потока $\Lambda_{1}$ и с вероятностью $p_{2}=1-p_{1}$ - точкой потока $\Lambda_{2}$ (рис. 2). Известно [5], что потоки $\Lambda_{1}, \Lambda_{2}$ также являются пуассоновскими с интенсивностями $\lambda p_{1}, \lambda p_{2}$ соответственно.

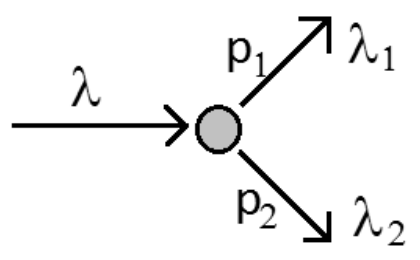

Рис. 2. Вероятностное раздвоение пуассоновского потока

Теорема 1. Потоки $\Lambda_{1}, \Lambda_{2}$ независимы.

Доказательство. Возьмем произвольный отрезок $[t, t+T], t \geq 0, T>0$, и обозначим $n, n_{1}, n_{2}$ количество точек потоков $\Lambda, \Lambda_{1}, \Lambda_{2}$ на этом отрезке. Вычислим вероятность

$$
\begin{gathered}
P\left(n_{1}=k_{1}, n_{2}=k_{2}\right)=P\left(n=k_{1}+k_{2}\right) C_{k_{1}+k_{2}}^{k_{1}} p_{1}^{k_{1}} p_{2}^{k_{2}}=\frac{\mathrm{e}^{-\lambda T}(\lambda T)^{k_{1}+k_{2}}}{\left(k_{1}+k_{2}\right) !} \frac{\left(k_{1}+k_{2}\right) !}{k_{1} ! k_{2} !} p_{1}^{k_{1}} p_{2}^{k_{2}}= \\
=\mathrm{e}^{-\lambda T p_{1}} \frac{\left(\lambda T p_{1}\right)^{k_{1}}}{k_{1} !} \cdot \mathrm{e}^{-\lambda T p_{2}} \frac{\left(\lambda T p_{2}\right)^{k_{2}}}{k_{2} !}=P\left(n_{1}=k_{1}\right) \cdot P\left(n_{2}=k_{2}\right) .
\end{gathered}
$$

Пусть теперь отрезки $\left[t^{(1)}, t^{(1)}+T^{(1)}\right],\left[t^{(2)}, t^{(2)}+T^{(2)}\right]$ не пересекаются. Обозначим $n^{(i)}, n_{1}^{(i)}, n_{2}^{(i)}$ количество точек потоков $\Lambda, \Lambda_{1}, \Lambda_{2}$ на отрезке $\left[t^{(i)}, t^{(i)}+T^{(i)}\right], i=1,2$. Докажем независимость случайных величин $n_{1}^{(1)}, n_{2}^{(2)}$. Для этого, полагая $k_{2}^{(1)}=k^{(1)}-k_{1}^{(1)}, k_{1}^{(2)}=k^{(2)}-k_{2}^{(2)}$, вычислим вероятность

$$
\begin{gathered}
P\left(n_{1}^{(1)}=k_{1}^{(1)}, n_{2}^{(2)}=k_{2}^{(2)}\right)=\sum_{k^{(1)} \geq k_{1}^{(1)}, k^{(2)} \geq k_{2}^{(2)}} P\left(n^{(1)}=k^{(1)}, n^{(2)}=k^{(2)}\right) C_{k_{1}^{k^{(1)}}}^{k_{1}^{(1)}} p_{2}^{k_{2}^{(1)}} C_{k_{1}^{(2)}}^{k_{1}^{(2)}} p_{1}^{k_{1}^{(2)}} p_{2}^{k_{2}^{(2)}}= \\
=\sum_{k^{(1)} \geq k_{1}^{(1)}, k^{(2)} \geq k_{2}^{(2)}} P\left(n^{(1)}=k^{(1)}\right) P\left(n^{(2)}=k^{(2)}\right) C_{k^{(1)}}^{k_{1}^{(1)}} p_{1}^{k_{1}^{(1)}} p_{2}^{k_{2}^{(1)}} C_{k_{1}^{(2)}}^{k_{1}^{(2)}} p_{1}^{k_{1}^{(2)}} p_{2}^{k_{2}^{(2)}}= \\
=\sum_{k^{(1)} \geq k_{1}^{(1)}, k^{(2)} \geq k_{2}^{(2)}} \mathrm{e}^{-\lambda T^{(1)}} \frac{\left(\lambda T^{(1)}\right)^{k^{(1)}}}{k^{(1)} !} \mathrm{e}^{-\lambda T^{(2)}} \frac{\left(\lambda T^{(2)}\right)^{k^{(2)}}}{k^{(2)} !} \frac{k^{(1)} !}{k_{1}^{(1)} ! k_{2}^{(1)} !} p_{1}^{k_{1}^{(1)}} p_{2}^{k_{2}^{(1)}} \frac{k^{(2)} !}{k_{1}^{(2)} ! k_{2}^{(2)} !} p_{1}^{k_{1}^{(2)}} p_{2}^{k_{2}^{(2)}}= \\
=\mathrm{e}^{-\lambda T^{(1)} p_{1}} \frac{\left(\lambda T^{(1)} p_{1}\right)^{k_{1}^{(1)}}}{k_{1}^{(1)} !} \mathrm{e}^{-\lambda T^{(2)} p_{2}} \frac{\left(\lambda T^{(2)} p_{2}\right)^{k_{2}^{(2)}}}{k_{2}^{(2)} !} \sum_{k_{2}^{(1)} \geq 0} \mathrm{e}^{-\lambda T^{(1)} p_{2}} \frac{\left(\lambda T^{(1)} p_{2}\right)^{k_{2}^{(1)}}}{k_{2}^{(1)} !} \sum_{k_{1}^{(2)} \geq 0} \mathrm{e}^{-\lambda T^{(2)} p_{1}} \frac{\left(\lambda T^{(2)} p_{1}\right)^{k_{1}^{(2)}}}{k_{1}^{(2) !}}= \\
=\mathrm{e}^{-\lambda T^{(1)} p_{1}} \frac{\left(\lambda T^{(1)} p_{1}\right)^{k_{1}^{(1)}}}{k_{1}^{(1)} !} \mathrm{e}^{-\lambda T^{(2)} p_{2}} \frac{\left(\lambda T^{(2)} p_{2}\right)^{k_{2}^{(2)}}}{k_{2}^{(2) !}}=P\left(n_{1}^{(1)}=k_{1}^{(1)}\right) P\left(n_{2}^{(2)}=k_{2}^{(2)}\right) .
\end{gathered}
$$


Таким образом, пары случайных величин $\left(n_{1}^{(1)}, n_{2}^{(1)}\right),\left(n_{1}^{(2)}, n_{2}^{(2)}\right),\left(n_{1}^{(1)}, n_{2}^{(2)}\right),\left(n_{2}^{(1)}, n_{1}^{(2)}\right)$ состоят из независимых компонент. Следовательно, пуассоновские потоки $\Lambda_{1}, \Lambda_{2}$ независимы.

Замечание 1. Утверждение теоремы 1 распространяется на случай, когда пуассоновский поток $\Lambda$ делится на $l>1$ пуассоновских потоков с помощью вероятностного просеивания, при котором каждая точка потока $\Lambda$ с вероятностью $p_{j}>0$ попадает в поток $\Lambda_{j}, j=1, \ldots, l, \sum_{j=1}^{l} p_{j}=1$.

\section{2. Независимость конечного числа потоков сети}

На множестве вершин ориентированного дерева $D$ (в каждую вершину которого входит только одно направленное ребро) определим отношение частичного порядка $u_{1} \succ u_{2}$, если в дереве $D$ существует путь из вершины $u_{1}$ в вершину $u_{2}$ (здесь $u \succ u$ ). Сопоставим каждой вершине $u$ дерева $D$ множества вершин $P(u)=\{v: u \succ v\}, Q(u)=\{v: v \succ u\} \cup P(u)$.

Теорема 2. Предположим, что вершины $u_{1}, \ldots, u_{k}$ дерева $D$ таковы, что множества $P\left(u_{1}\right), \ldots, P\left(u_{k}\right) \quad$ не пересекаются. Тогда для любых вершин $v_{1} \in P\left(u_{1}\right), \ldots, v_{k} \in P\left(u_{k}\right)$ потоки $\Lambda\left(v_{1}\right), \ldots, \Lambda\left(v_{k}\right)$, входящие в вершины $v_{1}, \ldots, v_{k}$, соответственно, независимы.

Доказательство. Из определения дерева $D$ следует, что в нем существует вершина $u$ такая, что непересекающиеся множества $P\left(u_{1}\right), \ldots, P\left(u_{k}\right)$ содержатся в множестве $P(u)$. В качестве $u$ можно взять, например, корень дерева. Следовательно, в силу теоремы 1 и замечания 1 потоки, выходящие из вершины $u$, независимы. Отсюда автоматически следует независимость потоков $\Lambda\left(v_{1}\right), \ldots, \Lambda\left(v_{k}\right)$.

Всюду далее без ограничения общности будем предполагать, что любая вершина дерева $D$ за исключением листьев имеет отвлетвление (из нее выходит несколько направленных ребер, рис. 3). Построим алгоритм выделения кластеров на множестве вершин дерева $D$. Пусть в дереве $D$ зафиксирована вершина $u_{1}$, не совпадающая с корневой вершиной, и определены соответствующие ей множества $P\left(u_{1}\right), Q\left(u_{1}\right), S_{1}=Q\left(u_{1}\right)$. Если на шаге $i \geq 1$ заданы $u_{i}, S_{i}$, то возьмем вершину $u_{i+1} \notin S_{i}$ и положим $S_{i+1}=S_{i} \cup Q\left(u_{i}\right)$. В силу конечности числа вершин в дереве $D$ эта процедура закончится на некотором шаге $l \geq 1$ построением вершин $u_{1}, \ldots, u_{i}$ и соответствующих им множеств $S_{1}, \ldots, S_{l}$.

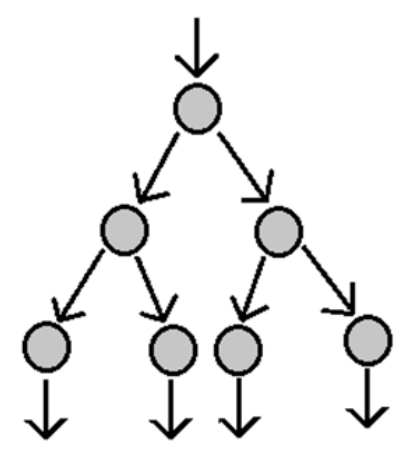

Рис. 3. Дерево с ответвлениями

Теорема 3. Множества вершин $P\left(u_{1}\right), \ldots, P\left(u_{i}\right)$ в дереве $D$ не пересекаются.

Доказательство. Пусть на шаге $i<l$ заданы вершины $u_{1}, \ldots, u_{i}$ и соответствующие им множества $S_{1}, \ldots, S_{i} ; P\left(u_{1}\right), \ldots, P\left(u_{i}\right)$. Тогда на шаге $i+1$ выполняется соотношение $u_{i+1} \notin S_{i}$ и, значит, множество $P\left(u_{i+1}\right)$ не пересекается с множествами $P\left(u_{1}\right), \ldots, P\left(u_{i}\right)$. В противном случае существуют 
такие $k, 1 \leq k \leq i, v \in P\left(u_{k}\right)$, что $v \in P\left(u_{i+1}\right)$. Следовательно, $u_{i+1} \succ v, u_{k} \succ v, u_{i+1} \neq u_{k}$ и, значит, $D$ не является ориентированным деревом.

Обозначим $m$ число ребер, выходящих из корневой вершины дерева $D$ и попадающих в вершины $v_{1}, \ldots, v_{m}$, и положим $M$ число листьев этого дерева.

Теорема 4 Справедливо неравенство

$$
m \leq l \leq M .
$$

Доказательство. Обозначим $V_{1}, \ldots, V_{m}$ наборы вершин дерева $D$, удовлетворяющие соотношениям $V_{j}=P\left(v_{j}\right), j=1, \ldots, m$. Из определения множеств $V_{1}, \ldots, V_{m}$ следует, что они не пересекаются, а их объединение совпадает с множеством всех вершин дерева $D$ за исключением корневой вершины. Поэтому для любого $j, 1 \leq j \leq m$, существует такое $i, 1 \leq i \leq l$, что $P\left(u_{i}\right) \subseteq P\left(v_{j}\right)$. В противном случае множество $S_{l}$ не будет совпадать с множеством вершин дерева $D$, что противоречит определению $S_{l}$. Следовательно, справедливо неравенство $m \leq l$. По определению объединение $\bigcup_{i=1}^{l} P\left(u_{i}\right)$ непересекающихся в силу теоремы 3 множеств $P\left(u_{1}\right), \ldots, P\left(u_{l}\right)$ содержит все листья ориентированного дерева $D$. Следовательно, $l \leq M$.

Замечание 2. Рекуррентная процедура, когда на каждом шаге выбирается вершина, в которую входит ребро-стрелка из корневой вершины, приводит к равенству $m=l$. Рекуррентная процедура, в которой на каждом шаге выбирается очередной лист дерева $D$, приводит к равенству $l=M$.

Замечание 3. Предположим, что вершины $u_{1}, \ldots, u_{k}$ дерева $D$ таковы, что множества $P\left(u_{1}\right), \ldots, P\left(u_{k}\right)$ не пересекаются и объединение множеств $\bigcup_{i=1}^{k} Q\left(u_{i}\right)$ совпадает с множеством вершин дерева $D$. Тогда описанная выше рекуррентная процедура может быть реализована с использованием этого набора вершин (рис. 4).
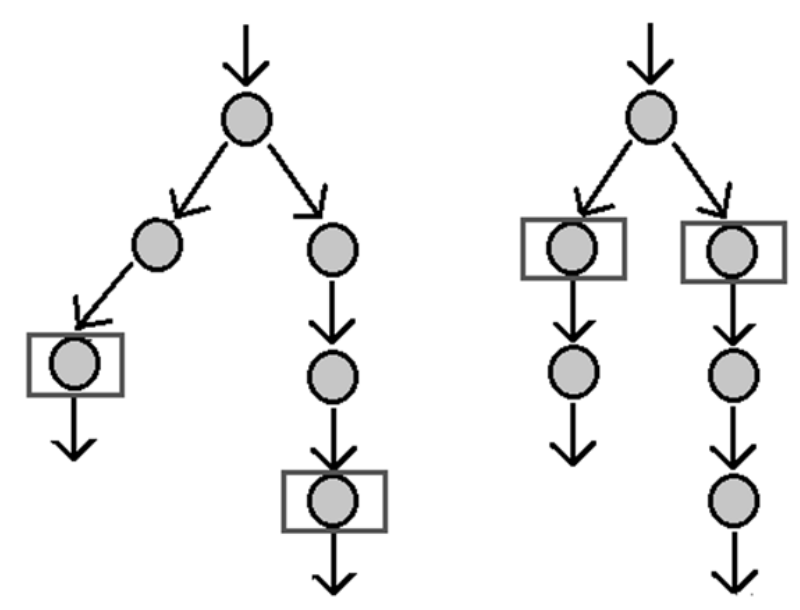

Рис. 4. Примеры совокупностей узлов с независимыми потоками

\section{Заключение}

Теоремы 2-4 справедливы для сетей, представляемых ориентированными деревьями общего вида без требования наличия отвлетвления в каждой вершине. Полученные в работе результаты можно распространить на сети, в каждом узле которых содержится конечное число каналов. От них возможен переход к сетям с бесконечным числом каналов путем выделения синергетических эффектов. В случае непуассоновского входного потока можно использовать результаты работы [7] для асимптотического анализа числа занятых каналов в каждом узле сети. 


\section{ЛИТЕРАТУРА}

1. Nobel R.D., Tijms H.C. Optimal control for an MX/G/1 queue with two service modes // European Journal of Operational Research. 1999. Elsevier. V. 113(3). P. 610-619.

2. Nobel R.D., Tijms H.C. Waiting-time probabilities in the "M/G/1" retrial queue // Statistica Neerlandica. 2006. Netherlands Society for Statistics and Operations Research. V. 60 (1). P. 73-78.

3. Жидкова Л.А., Моисеева С.П. Исследование системы параллельного обслуживания кратных заявок простейшего потока // Вестник Томского государственного университета. Управление, вычислительная техника и информатика. 2011. № 4 (17). C. 49-54.

4. Жидкова Л.А., Моисеева С.П. Математическая модель потоков покупателей двухпродуктовой торговой компании в виде системы массового обслуживания с повторными обращениями к блокам // Известия Томского политехнического университета. 2013. Т. 322, № 6. С. 5-9.

5. Хинчин А.Я. Работы по математической теории массового обслуживания. М. : Физматлит, 1963.

6. Burke P.J. The output of a queuing system // Operations Research. 1956. V. 4. P. 699-704.

7. Моисеев А.Н., Назаров А.А. Бесконечнолинейные системы и сети массового обслуживания. Томск : Изд-во НТЛ, 2015.

Цициашвили Гурами Шалвович, д-р физ.-мат. наук, професcop. E-mail: guram@iam.dvo.ru Институт прикладной математики ДВО РАН,

Дальневосточный федеральный университет, г. Владивосток

Поступила в редакцию 28 апреля 2016 г.

Tsitsiashvili Gurami Sh. (Institute for Applied Mathematics of Far Eastern Branch of RAS, Far Eastern Federal University. Vladivostok).

Poisson flows in systems with retrial queues.

Keywords: systems with retrial queues; Poisson flows; independence of flows.

\section{DOI: $10.17223 / 19988605 / 37 / 9$}

Queuing systems with retrial queues represent large interest in manifold applications of the queuing theory. Especially last years it is connected with infinite server queuing systems and retrial queues. These models may be considered in terms of queuing networks which have a structure of directed tree. An analysis of such networks may be based on the Burke theorem and the graph theory concepts in a definition of independence of random flows. In this paper an attempt to solve this problem in the most general terms is realized.

Consider infinite server queuing system with Poisson input flow and exponentially distributed service times. Assume that each customer after a service may be served iteratively. The different protokols of functioning for such system may be realized. For example, a distribution of repeated service time may differ from a distribution of initial service time, a number of repeated service may be different and so on

The most general model of such system is a model of queuing network in which each node has infinite number of servers with exponential distribution of service times. Such network is described by a directed tree $D$ in which any edge is directed from a tree root $r$ (a node which have not incoming edges) to its leaves (nodes which have not outgoing edges). On service finishing a customer may be directed to some nodes where its outcoming edges show. Natural generalization of this model is based on an asumption that service times have hyperexponential distributions (probability mixtures of exponential distributions). This asumption does not change a view of this network in which transitions between nodes are discribed by oriented tree also.

From the Burke theorem we have that if the network is in a stationary regime then its outcomig and incoming flows of each node are Poisson. But a problem is on dependent or independent for these flows. This problem is solved as follows.

Consider now Poisson point flow $\Lambda=\left\{0 \leq t_{1} \leq t_{2} \leq \ldots\right\}$ with the intensity $\lambda$. Assume that each point of the flow $\Lambda$ independently with a probability $p_{1}, 0<p_{1}<1$, becomes a point of a flow $\Lambda_{1}$ and with a probability $p_{2}=1-p_{1}-$ a point of a flow $\Lambda_{2}$ It is well known that the flows $\Lambda_{1}, \Lambda_{2}$ are Poisson flows also with intensities $\lambda p_{1}, \lambda p_{2}$, accordingly.

Theorem 1. Flows $\Lambda_{1}, \Lambda_{2}$ are independent.

On a set of the directed tree $D$ (in which each node has a single incoming edge) define a relation of partial order $u_{1} \succ u_{2}$, if in the tree $D$ there is a way from the node to the node $u_{2}$ (here $u \succ u$ ). Contrast each node $u$ of the tree $D$ the nodes sets $P(u)=\{v: u \succ v\}, Q(u)=\{v: v \succ u\} \cup P(u)$.

Theorem 2. Assume that nodes $u_{1}, \ldots, u_{k}$ of the tree $D$ satisfy the condition that the sets $P\left(u_{1}\right), \ldots, P\left(u_{k}\right)$ do not intersect.Then for any nodes $v_{1} \in P\left(u_{1}\right), \ldots, v_{k} \in P\left(u_{k}\right)$ flows $\Lambda\left(v_{1}\right), \ldots, \Lambda\left(v_{k}\right)$, incoming to the nodes $v_{1}, \ldots, v_{k}$, appropriately are independent.

Obtained results may be spread onto networks with final number of servers in their nodes and to transit from these networks to networks with infinite nimber of servers by analyzing synergetic effects. If input network flow is recurrent then it is possible to use results on asymptotic analysis of numbers of occupied servers in network nodes.

\section{REFERENCES}

1. Nobel, R.D. \& Tijms, H.C. (1999) Optimal control for an MX/G/1 queue with two service modes. European Journal of Operational Research. 113(3). pp. 610-619. DOI: 10.1007/978-81-322-1680-3_6. 814 
2. Nobel, R.D. \& Tijms, H.C. (2006) Waiting-time probabilities in the "M/G/1" retrial queue. Statistica Neerlandica. Netherlands Society for Statistics and Operations Research. 60(1). pp. 73-78. DOI: 10.1111/j.1467-9574.2006.00312.x

3. Zhidkova, L.A. \& Moiseeva, S.P. (2011) Investigation of parallel serving system with simplest flow of fold customers. Vestnik Tomskogo gosudarstvennogo universiteta. Upravlenie, vychislitel'naya tekhnika i informatika - Tomsk State University Journal of Control and Computer Science. 4(17). pp. 49-54. (In Russian).

4. Zhidkova, L.A. \& Moiseeva, S.P. (2013) Mathematical model of customer flows of two product trade company as queuing system with retrial queues. Izvestiya Tomskogo politekhnicheskogo universiteta - Bulletin of the Tomsk Polytechnic University. 322(6). pp. 5-9. (In Russian).

5. Khinchin, A.Ya. (1963) Raboty po matematicheskoy teorii massovogo obsluzhivaniya [Researchs on mathematical queuing theory]. Moscow: Phyzmatgiz.

6. Burke, P.J. (1956) The output of a queuing system. Operations Research. 4. pp. 699-704. DOI: 10.1287/opre.4.6.699

7. Moiseev, A.N. \& Nazarov, A.A. (2015) Beskonechnolineynye sistemy i seti massovogo obsluzhivaniya [Infinite server queuing systems and networks]. Tomsk. NTL. 